Research article

\title{
Diagnostic value of anti-topoisomerase I antibodies in a large monocentric cohort
}

\author{
Katharina Hanke1, Cornelia Dähnrich², Claudia S Brückner ${ }^{1}$, Dörte Huscher ${ }^{3}$, Mike Becker ${ }^{1}$, \\ Anthonina Jansen², Wolfgang Meyer ${ }^{2}$, Karl Egerer ${ }^{1}$, Falk Hiepe ${ }^{1}$, Gerd R Burmester ${ }^{1}$, \\ Wolfgang Schlumberger ${ }^{2}$ and Gabriela Riemekasten ${ }^{1}$
}

\author{
1Department of Rheumatology and Clinical Immunology, Charité Universitätsmedizin Berlin, Charitéplatz 1, Berlin 10117, Germany \\ 2EUROIMMUN Medizinische Labordiagnostika AG, Seekamp 31, Lübeck 23560, Germany \\ ${ }^{3}$ German Rheumatism Research Centre, Charitéplatz 1, Berlin 10117, Germany
}

Corresponding author: Gabriela Riemekasten, gabriela.riemekasten@charite.de

Received: 8 Sep 2008 Revisions requested: 20 Oct 2008 Revisions received: 21 Jan 2009 Accepted: 21 Feb 2009 Published: 21 Feb 2009

Arthritis Research \& Therapy 2009, 11:R28 (doi:10.1186/ar2622)

This article is online at: http://arthritis-research.com/content/11/1/R28

(C) 2009 Hanke et al.; licensee BioMed Central Ltd.

This is an open access article distributed under the terms of the Creative Commons Attribution License (http://creativecommons.org/licenses/by/2.0), which permits unrestricted use, distribution, and reproduction in any medium, provided the original work is properly cited.

\begin{abstract}
Introduction In the present study, the detection of antitopoisomerase I (anti-topo I) autoantibodies was evaluated for diagnosis and risk assessment of systemic sclerosis (SSc) patients in a well characterized large monocentric cohort.

Methods Sera from patients with SSc (diffuse $n=96$, limited $n$ $=113$ ), from patients with overlap syndromes $(n=51)$, from patients with other diseases associated with SSc $(n=20)$, as well as from disease controls $(n=487)$ were analysed for the presence of anti-topo I antibodies by line immunoblot assay and ELISA. Assessment of organ manifestations was performed as proposed by the European Scleroderma Trial and Research network.

Results The applied test systems for the detection of anti-topo I antibodies revealed a diagnostic sensitivity for SSc of approximately $24 \%$ and a diagnostic specificity of at least

$99.6 \%$. The sensitivity to identify patients with diffuse SSc amounted to $60 \%$. Patients with anti-topo I antibodies showed a higher burden of skin and lung fibrosis, contractures, electrocardiogram changes, as well as digital ulcers and had more active disease than antibody-negative patients. Signal strengths correlated only weakly with disease activity, with modified Rodnan skin score, with predicted forced vital capacity, and with predicted diffusion capacity levels $(P=0.01$, $\rho=0.234, \rho=0.413, \rho=-0.215, \rho=-0.219)$. High signal intensities were associated with an increased mortality in diffuse SSc patients $(P=0.003)$.

Conclusions Diagnosis and risk assessment of SSc patients can be supported by the detection of anti-topo I antibodies. Signal intensities as obtained by line immunoblot assay or ELISA can be used as a surrogate marker for fibrosis, active disease and worse prognosis.
\end{abstract}

\section{Introduction}

Systemic sclerosis (SSc) is a rare and heterogeneous disease with different disease subsets. Its outcome may vary from mild to very severe, life-threatening disease rapidly leading to death. The detection of autoantibodies, especially directed towards topoisomerase I (anti-topo I), can help to identify patients at risk for progressive and severe disease and to classify them according to their disposition for certain clinical manifestations [1-6]. Depending on the studies, however, antibody frequencies varied between 14\% [2] and 70\% [4].
Several commercially available test systems can be used for the detection of anti-topo I antibodies. Among these systems, efficient monospecific methods - such as ELISAs and line immunoblot assays (LIAs) for single-parameter or profile analysis - have been established in clinical laboratory practice during past years, allowing examination of a large number of sera in a rapid approach at high sensitivity and at high specificity [710]. 
The precise clinical characterization of the patients and their assessment are crucial for the evaluation of a new assay or of a potential biomarker such as anti-topo I antibodies as well as for the comparison with other studies. As shown before, differences in the frequencies of anti-topo I antibodies in different studies might be caused by ethnic background $[1,11]$ or by different classification systems of SSc [1,2,12-14]. Most studies have used the American College of Rheumatology classification criteria $[1,2,13]$; however, there are some limitations especially for early and probably very late cases, as these criteria were originally not intended to be used as a diagnostic tool. Other studies have divided their patients into diffuse, limited, and intermediate SSc patients or have included overlap syndromes $[13,14]$. In recent years, the LeRoy criteria for classification have been widely used. In this classification system, however, the presence of anti-topo I antibodies is often associated with diffuse SSc [15].

Most studies have shown that anti-topo I-positive patients suffer more frequently from severe lung and skin fibrosis $[4,6,13,16]$. Several studies have identified further associations not confirmed by others while using different assessment strategies to define organ involvement or disease activity $[17,18]$. Additionally, the interval between the detection of antibodies and the clinical assessment often remains unspecified [1]. National and multinational networks such as the European Systemic Sclerosis Trial and Research (EUSTAR) network or the German Network of Systemic Sclerosis (DNSS) addressed the standardization in the assessment and classification of patients. Despite these efforts, both intraobserver variability and interobserver variability are still significant, especially when multicentric studies are performed [19].

In the present cross-sectional monocentric study, we analysed a large cohort of genuine SSc patients, patients with diseases related to SSc as well as numerous disease controls for the presence and diagnostic impact of anti-topo I antibodies detected by means of commercially available, monospecific LIA and ELISA. In order to minimize limitations of former studies, clinical data were assessed simultaneously to antibody detection by a standardized procedure with only a limited number of investigators.

\section{Materials and methods Classification of patients}

Sera from 280 consecutive patients with SSc were tested for the presence of anti-topo I antibodies. As disease controls we included serum samples from patients with myositis $(n=26)$, from patients with systemic lupus erythematosus $(n=208)$, from patients with Sjögren's syndrome $(n=88)$ and from patients with rheumatoid arthritis $(n=165)$. All patients were diagnosed at the Charité University of Medicine (Berlin, Germany).
Classification of SSc patients was performed before starting the study based on the maximal skin involvement during the disease course, on the basis of the LeRoy criteria and according to the EUSTAR and DNSS criteria [20]. Briefly, SSc patients were classified as having either limited SSc or diffuse SSc depending on the distribution of skin sclerosis below or above the elbows, knees or clavicles [15]. Once classified as having diffuse SSc, the patient remains classified as having diffuse SSc. Characteristics of SSc patients are presented in Table 1.

Overlap syndrome was defined as a disease occurring with clinical aspects of SSc (according to the American College of Rheumatology criteria) or with the main symptoms of SSc simultaneously with those of other connective tissue diseases/ other autoimmune diseases, such as dermatomyositis, Sjögren's syndrome or systemic lupus erythematosus. These patients are mostly positive for anti-U1-RNP antibodies or antiPM-Scl antibodies [21,22]. SSc sine scleroderma was defined by the lack of skin alterations in the presence of other SSc symptoms [23]. Undifferentiated connective tissue disease with scleroderma features was defined as positive Raynaud's phenomenon and at least one further feature of SSc and/or detectable scleroderma-associated autoantibodies [24].

\section{Assessment of systemic sclerosis patients}

With the exception of the subclassification of SSc patients, clinical data were collected simultaneously at the time sera were obtained for the detection of anti-topo I antibodies. Most patients were assessed by one investigator (GR), and the second investigator involved (CB) was instructed by the first investigator. Both investigators participated in several training programs of EUSTAR and DNSS for the assessment of SSc patients.

The modified Rodnan Skin Score (mRSS) was used for the evaluation of fibrotic skin changes $[25,26]$. Cardiac involvement was defined by the presence of two of the following symptoms: diastolic dysfunction, conduction abnormalities, cardiomyopathy, or reduced ejection fraction unrelated to other diseases, valvular changes such as tricuspidal insufficiency not explained for by other causes than SSc, or pericarditis. Conduction blocks and signs of atrial and ventricular hypertrophy related to SSc were summarized as electrocardiogram changes. Disease activity was obtained using the EUSTAR activity index [17]. Pulmonary arterial hypertension was defined by a mean pulmonary arterial pressure of $25 \mathrm{mmHg}$ at rest and $30 \mathrm{mmHg}$ at exercise when assessed by right heart catheterization or by the presence of pulmonary artery systolic pressure $\geq 40 \mathrm{mmHg}$ as detected by echocardiography and signs of right heart failure. Pulmonary fibrosis was diagnosed by chest radiogram and/or by high-resolution computed tomography scans. Lung function was assessed by the predicted forced vital capacity (FVC) and the predicted diffusion 
Clinical and demographic characteristics of the systemic sclerosis (SSc) cohort (Charité University)

\begin{tabular}{|c|c|c|c|c|c|c|c|}
\hline & Diffuse SSc & Limited SSc & $\begin{array}{l}\text { SSc sine } \\
\text { scleroderma }\end{array}$ & Overlap & $\begin{array}{l}\text { Mixed } \\
\text { connective } \\
\text { tissue disease }\end{array}$ & $\begin{array}{l}\text { Undifferentiated } \\
\text { connective tissue } \\
\text { disease }\end{array}$ & All patients \\
\hline $\begin{array}{l}\text { Anti- } \\
\text { topoisomerase I } \\
\text { (LIA) }\end{array}$ & $58(60.4)$ & $7(6.2)$ & $1(25.0)$ & $1(2.6)$ & 0 & 0 & $67(23.9)$ \\
\hline $\begin{array}{l}\text { Anti- } \\
\text { topoisomerase I } \\
\text { (ELISA) }\end{array}$ & $57(59.4)$ & $6(5.3)$ & $1(25.0)$ & $1(2.6)$ & 0 & 0 & $65(23.2)$ \\
\hline Number & 96 & 113 & 4 & 38 & 13 & 16 & 280 \\
\hline Age (years) & $53 \pm 14.5$ & $60 \pm 11.3$ & $54 \pm 12.7$ & $52 \pm 13.3$ & $52 \pm 13.5$ & $56 \pm 10.7$ & $56 \pm 13.2$ \\
\hline $\begin{array}{l}\text { Duration of } \\
\text { Raynaud's } \\
\text { phenomenon } \\
\text { (years) }\end{array}$ & $9 \pm 10.6$ & $14 \pm 13.6$ & $18 \pm 18.47$ & $8 \pm 7.4$ & $16 \pm 10.4$ & $11 \pm 9.3$ & $12 \pm 11.6$ \\
\hline $\begin{array}{l}\text { Duration of non- } \\
\text { Raynaud's } \\
\text { phenomenon } \\
\text { symptoms }\end{array}$ & $7.8 \pm 1.6$ & $9.5 \pm 1.39$ & $6 \pm 10.8$ & $8.8 \pm 2.2$ & $9.1 \pm 5.5$ & $9.3 \pm 6.3$ & $8.7 \pm 7.6$ \\
\hline $\begin{array}{l}\text { Duration since } \\
\text { disease diagnosis } \\
\text { (years) }\end{array}$ & $6 \pm 7.38$ & $8 \pm 7.4$ & $3 \pm 2.6$ & $6 \pm 5.17$ & $9.1 \pm 5.5$ & $7 \pm 7.28$ & $7 \pm 7.38$ \\
\hline Female/male & $79 / 17$ & $105 / 8$ & $2 / 2$ & $28 / 10$ & $13 / 0$ & $16 / 0$ & $243 / 37$ \\
\hline mRSS & $13.2 \pm 9.6$ & $5.2 \pm 4.2$ & $1.5 \pm 1.1$ & $6.7 \pm 7.5$ & $1.6 \pm 1.8$ & $0.8 \pm 1.1$ & $7.6 \pm 8.0$ \\
\hline Digital ulcers & $52(54.2)$ & 38 (33.6) & $2(50)$ & $14(36.8)$ & $4(30.8)$ & $2(12.5)$ & $112(40.0)$ \\
\hline Lung fibrosis & $57(59.4)$ & $16(14.2)$ & $3(75)$ & $16(42.1)$ & $4(30.8)$ & $2(12.5)$ & $98(35.0)$ \\
\hline $\begin{array}{l}\text { DLCO by a single } \\
\text { breath (\%) }\end{array}$ & $64.9 \pm 21.9$ & $76,8 \pm 17.5$ & $56.6 \pm 12.8$ & $64.2 \pm 22.4$ & $75.3 \pm 17.8$ & $75.4 \pm 17.1$ & $70.5 \pm 20.5$ \\
\hline Mean FVC (\%) & $81.7 \pm 18.93$ & $96.7 \pm 15.2$ & $87.5 \pm 30.7$ & $84.0 \pm 19.2$ & $92.9 \pm 23.5$ & $92 \pm 18.0$ & $89,2 \pm 19$ \\
\hline Contractures & 78 (81.3) & $62(54.9)$ & $2(50)$ & $23(60.5)$ & $4(30.8)$ & $3(18.8)$ & $172(61.4)$ \\
\hline $\begin{array}{l}\text { Pulmonary arterial } \\
\text { hypertension }\end{array}$ & $22(22.9)$ & $22(19.5)$ & $2(50)$ & $9(23.7)$ & $4(30.8)$ & $1(6.3)$ & $60(21.4)$ \\
\hline $\begin{array}{l}\text { Renal } \\
\text { involvement }\end{array}$ & $17(17.7)$ & $22(19.5)$ & $1(25)$ & $10(26.3)$ & $4(30.8)$ & $2(12.5)$ & $56(20)$ \\
\hline Renal crisis & $10(10.4)$ & $3(2.7), n=112$ & $1(25)$ & $1(2.6)$ & 0 & $1(6.3)$ & $\begin{array}{l}16(5.7), n= \\
279\end{array}$ \\
\hline $\begin{array}{l}\text { Cardiac } \\
\text { involvement }\end{array}$ & $47(49.0)$ & $35(31.0)$ & $3(75)$ & $19(50)$ & $6(46.2)$ & $4(25)$ & $117(40.7)$ \\
\hline Skin involvement & 94 (98.9) & 105 (92.9) & $3(75)$ & $32(84.2)$ & $7(53.8)$ & $7(43.8)$ & $248(88.9)$ \\
\hline $\begin{array}{l}\text { Raynaud's } \\
\text { phenomenon }\end{array}$ & 95 (99) & $110(97.3)$ & $4(100)$ & $38(100)$ & 12 (92.3) & $13(81.3)$ & $272(97.1)$ \\
\hline
\end{tabular}

Data presented as mean \pm standard deviation or $n(\%)$. DLCO, predicted diffusion capacity; FVC, predicted forced vital capacity; LIA, line immunoblot assay; mRSS, modified Rodnan Skin Score.

capacity (DLCO) by a single breath method. Renal involvement was diagnosed by present or past renal crisis or impaired kidney function unexplained by other causes. Digital ulcers were defined as a loss of both epidermis and dermis in an area of at least $2 \mathrm{~mm}$ diameter at the distal phalanx of fingers.

Patients were included in the study between January 2004 and May 2007. For a prospective survival analysis, patients were observed for a mean period of 24.6 months (18 to 52 months). To assess cumulative survival data from the time point of diagnosis, primary care doctors or patients were contacted in order to monitor survival and treatment in patients without regular appointments. 
The study was approved by the local ethical committee (EA1/ 013/705). Written informed consent was obtained from each patient.

\section{Antibody detection}

Sera from consecutive patients with SSc-related diseases as well as sera serving as disease controls were obtained simultaneously with the clinical assessment of the patients and were stored at $-20^{\circ} \mathrm{C}$. Anti-topo I antibodies were detected by commercially available, CE-certified monospecific LIA (AntiScl-70 EUROLine) and ELISA (Anti-Scl-70 ELISA) provided by EUROIMMUN AG (Lübeck, Germany). Blot strips and ELISA wells are coated with commercial Scl-70 antigen purified by affinity chromatography from bovine thymus ( $>90 \%$ purity). This antigen (molecular weight 75 to $82 \mathrm{kDa}$ ) represents a proteolytic degradation product of the predominant in vivo form of the enzyme DNA topoisomerase I (molecular weight $100 \mathrm{kDa}$ ); both the native and lower molecular weight forms have been shown to effectively bind autoantibodies against topoisomerase I [27]. Antigen reactivity of LIA and ELISA was verified using the human reference serum CDCANA \#9 (Center for Disease Control, Atlanta, GA, USA) and both test systems were validated thoroughly using well characterized positive and negative controls.

All analyses were performed according to the manufacturer's instructions and were carried out blindly by personnel unaware of the diagnosis and the clinical characteristics of the patients.

For the LIA, human sera were diluted 1:101 prior to use and antibody detection was performed using alkaline phosphatase-labelled goat anti-human lgG. Control sera were included in each assay. Incubated blot strips were digitalized using a flatbed scanner. The intensity of the bands was automatically evaluated by a computer program. Signal strengths above 6 units were considered positive, as recommended by the manufacturer.

With respect to the ELISA, all serum samples were analysed at a dilution of 1:201 in parallel with control sera. Peroxidaselabelled rabbit anti-human IgG served as the secondary antibody conjugate. For evaluation, data above the cut-off value of 20 units/ml were considered positive, complying with the manufacturer's recommendation.

\section{Statistical analysis}

The dataset was analysed using the SPSS V 15.0 statistical package (NASDAQ, Bloomingdale, IL 60108, USA) and the Microsoft calculation software Excel V 12 (2007; Microsoft corporation, USA). To identify associations between SSc symptoms and the occurrence of anti-topo I antibodies, chisquare tests, Mann-Whitney $U$ tests, and Wilcoxon signedrank tests were performed when appropriate. Spearman's rank correlation coefficient $(\rho)$ was applied to analyse possible cor- relations. For all tests, $P<0.05$ was considered statistically significant.

\section{Results \\ Diagnostic sensitivity and specificity of anti- topoisomerase I antibody detection and identification of SSc patients with diffuse disease}

Using the monocentric SSc cohort $(n=280)$ and four disease control cohorts $(n=487)$, the detection of anti-topo I antibodies revealed a diagnostic sensitivity for SSc of $23.9 \%$ (23.2\%) and a diagnostic specificity of $99.6 \%$ (99.8\%), as determined by LIA (ELISA) (Table 2). In general, a significant correlation was observed between LIA signal strengths and antibody levels as detected by ELISA $(P<0.0005, \rho=0.752$; Figure 1$)$.

Since the SSc cohort was rather heterogeneous, the frequency of anti-topo I antibodies was determined for the distinct patient groups and disease manifestations, resulting in sensitivities and specificities for the serological identification of these clinical characteristics with reference to the SSc cohort (Table 2 and Table 3 ). LIA analyses revealed the presence of anti-topo I antibodies in 58 (60.4\%) out of 96 patients with diffuse SSc. In contrast, only seven (6.2\%) out of 113 patients with limited SSc, just one among 38 patients with an overlap syndrome other than mixed connective tissue disease and only one out of four patients with SSc sine scleroderma were positive for anti-topo I antibodies. Identical results were obtained by means of ELISA, with the exception of only 57 (59.4\%) anti-topo I-positive patients with diffuse SSc and six positive results $(5.3 \%)$ in the group of limited SSc. Based on these data, detection of anti-topo I antibodies by means of LIA (ELISA) revealed a sensitivity for diffuse SSc of $60.4 \%$ $(59.4 \%)$ with a specificity of $95.1 \%(95.6 \%)$, referring to the cohort of 280 patients with SSc.

Moreover, LIA signal strengths were stronger in diffuse SSc patients compared with limited SSc patients. The mean signal intensity was 148.4 units (standard deviation $=4.4$ ) in diffuse SSc patients and 103.6 units (standard deviation $=24.5$ ) in limited SSc patients $(P<0.0005)$, supporting the quantitative data obtained by ELISA (data not shown). With the ELISA, patients with diffuse SSc had higher mean values (467.6 units $/ \mathrm{ml}$, standard deviation $=68.1$ ) when compared with patients with limited SSc (233.2 units $/ \mathrm{ml}$, standard deviation $=97.3, P<0.0005)$.

Owing to the substantial degree of diagnostic conformity between LIA and ELISA (reflected by the above presented data and the data not shown), only results obtained by LIA are presented in the following sections. 
Table 2

\begin{tabular}{llll}
\hline Sensitivity and specificity of anti-topoisomerase I antibodies & & & \\
\hline Panel & $n$ & Line immunoblot assay & ELISA \\
\hline Diffuse systemic sclerosis $^{\mathrm{a}}$ & 96 & $58(60.4 \%)$ & $57(59.4)$ \\
Limited systemic sclerosis $^{\mathrm{a}}$ & 113 & $7(6.2 \%)$ & $6(5.3 \%)$ \\
Systemic sclerosis sine scleroderma $^{\mathrm{a}}$ & 4 & $1(25 \%)$ & $1(25 \%)$ \\
Overlap $^{\mathrm{a}}$ & 38 & $1(2.6 \%)$ & $1(2.6 \%)$ \\
Mixed connective tissue disease $^{\mathrm{a}}$ & 13 & 0 & 0 \\
Undifferentiated connective tissue disease $^{\mathrm{a}}$ & 16 & 0 & 0 \\
$\quad$ Sensitivity & & $65(23.2 \%)$ \\
Myositis $^{\mathrm{b}}$ & 280 & $67(23.9 \%)$ & $0(100 \%)$ \\
Systemic lupus erythematosus $^{\mathrm{b}}$ & 26 & $0(100 \%)$ & $0(100 \%)$ \\
Sjögren's syndrome $^{\mathrm{b}}$ & 208 & $0(100 \%)$ & $0(100 \%)$ \\
Rheumatoid arthritis $^{\mathrm{b}}$ & 88 & $0(100 \%)$ & $1(99.4 \%)$ \\
\multicolumn{1}{c}{ Specificity } & 165 & $2(98.8 \%)$ & $1(99.8 \%)$
\end{tabular}

aData presented as number of patients positive in the respective test (percentage within the respective patient cohort). bData presented as number of patients positive in the respective test (percentage of negative results within the respective control cohort).

\section{Anti-topoisomerase I antibodies were associated with peripheral vascular complications but not with pulmonary arterial hypertension or renal crisis}

Patients with anti-topo I antibodies revealed a higher frequency of present or past digital ulcers (Table 3): $57 \%$ of the anti-topo I-positive patients suffered from present or past digital ulcers. In patients without anti-topo I antibodies, the prevalence of digital ulcers was significantly lower (32.2\%).

In contrast, there was no association between the presence of anti-topo I antibodies and pulmonary arterial hypertension or renal involvement including renal crisis. There was also no association between the presence of anti-topo I antibodies and neuropathies, heart (despite conduction disturbances) and gastrointestinal involvement, renal involvement, sicca syndrome, joint involvement, or any other clinical symptom assessed by the EUSTAR and DNSS core dataset (data not shown) $[16,28]$.

\section{Anti-topoisomerase I antibodies characterize patients with fibrotic SSc features and active disease}

Among the $\mathbf{2 8 0}$ patients with SSc, anti-topo I-positive patients were characterized by fibrotic signs of SSc. As detected by LIA, anti-topo I-positive patients had a significantly higher prevalence of lung fibrosis (68.6\%) when compared with the antitopo I-negative group (23.3\%). In addition, patients with antitopo I antibodies had a lower predicted FVC when compared with the anti-topo I-negative SSc group (Tables 1 and 3). Only $15.6 \%$ of the patients with a predicted FVC $>85 \%$ had antitopo I antibodies, but $55 \%$ of the patients with a FVC of $50 \%$ to $69 \%$ had anti-topo I antibodies (Figure 2). The association between the group of predictive FVC and the presence of antitopo I antibodies was significant $(P=0.006)$.
Similar results were obtained for the predicted DLCO by a single breath. Patients with anti-topo I antibodies had a lower predicted DLCO when compared with antibody-negative patients (Table 3). Of the patients with a predicted DLCO $>85 \%$, $15.5 \%$ were anti-topo I-positive; in contrast, the frequency of patients with anti-topo I antibodies increased to $43.8 \%$ in patients with DLCO $<35 \%$ (Figure 2). Again, the groups of DLCO levels were significantly related to the presence of antitopo I antibodies $(P<0.001)$. Patients with anti-topo I antibodies also had a higher mRSS compared with patients without

\section{Figure 1}

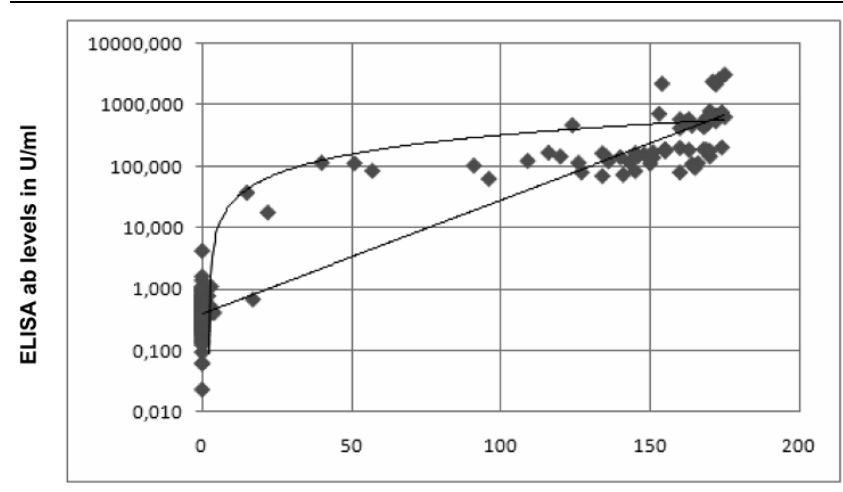

LIA signal strenths (U)

Line immunoblot assay signal strengths and logarithmized ELISA values to detect anti-topoisomerase I antibodies. Correlation between line immunoblot assay (LIA) signal strengths and logarithmized ELISA values for the detection of anti-topoisomerase I antibodies in our cohort of 280 patients. The logarithmic curve as indicated by the Spearman correlation coefficient reflects the correlation better than a linear correlation (as shown by the line). ab, antibody; $U$, units. 
Table 3

Anti-topoisomerase I antibody-positive versus antibody-negative patients in systemic sclerosis cohort assessed by line immunoblot assay

\begin{tabular}{|c|c|c|c|c|c|c|}
\hline \multirow[t]{2}{*}{ Disease manifestation } & \multirow{2}{*}{$\begin{array}{l}\text { Patients with disease } \\
\text { manifestation }\end{array}$} & \multicolumn{2}{|c|}{ Anti-topoisomerase I } & \multirow[t]{2}{*}{$P$ value } & \multirow[t]{2}{*}{ Sensitivity (\%) } & \multirow[t]{2}{*}{ Specificity (\%) } \\
\hline & & Positive $(n=67)$ & Negative $(n=213)$ & & & \\
\hline Digital ulcers & $112(40.0 \%)$ & $38(56.7 \%)$ & $74(34.7 \%)$ & 0.002 & 33.9 & 82.7 \\
\hline Lung fibrosis & $98(35.0 \%)$ & $46(68.6 \%)$ & $52(24.4 \%)$ & $<0.0005$ & 46.9 & 88.5 \\
\hline DLCO by a single breath & & $62.9 \pm 20.8$ & $72.9 \pm 19.9$ & $<0.0005$ & NA & NA \\
\hline Mean FVC & & $81.1 \pm 18.0$ & $91.8 \pm 18.7$ & $<0.0005$ & NA & NA \\
\hline $\begin{array}{l}\text { Pulmonary arterial } \\
\text { hypertension }\end{array}$ & $60(21.4 \%)$ & $17(25.4 \%)$ & $43(20.2 \%)$ & 0.39 & 28.3 & 77.3 \\
\hline Contractures & $162(57.9 \%)$ & $59(88.1 \%)$ & $113(53.1 \%)$ & $<0.0005$ & 34.3 & 92.6 \\
\hline Renal involvement & $56(20.0 \%)$ & $13(19.4 \%)$ & $43(20.2 \%)$ & 1 & 23.2 & 75.9 \\
\hline Renal crisis & $16(5.7 \%)$ & $6(9 \%)$ & $10(4.7 \%)$ & 0.23 & 37.5 & 76.8 \\
\hline Cardiac involvement & $114(40.7 \%)$ & $33(49.2 \%)$ & $81(38.0 \%)$ & 0.12 & 28.9 & 79.5 \\
\hline Conduction blocks & $69(24.6 \%)$ & $25(37.3 \%)$ & $44(20.7 \%)$ & 0.009 & 36.2 & 80.1 \\
\hline Electrocardiogram changes & $74(26.4 \%)$ & $27(40.3 \%)$ & $47(22.1 \%)$ & 0.007 & 36.5 & 80.4 \\
\hline mRSS & & $14.2 \pm 10.2$ & $5.57 \pm 5.8$ & $<0.0005$ & NA & NA \\
\hline Raynaud's phenomenon & $272(97.1 \%)$ & $67(100 \%)$ & $205(96.2 \%)$ & 0.2 & 24.6 & 100 \\
\hline Diffuse systemic sclerosis & $96(34.3 \%)$ & $58(86.6 \%)$ & $38(17.8 \%)$ & $<0.0005$ & 60.4 & 95.1 \\
\hline Mean disease activity & & 2.49 & 1.63 & $<0.0005$ & NA & NA \\
\hline
\end{tabular}

Data presented as $n(\%)$ or mean \pm standard deviation. DLCO, predicted diffusion capacity; FVC, predicted forced vital capacity; mRSS, modified Rodnan skin score; NA, not applicable.

anti-topo I antibodies (Table 3 ). Only $2.2 \%$ of patients with a mRSS between 0 and 3 had anti-topo I antibodies. In contrast, $61.4 \%$ of the patients with a mRSS $>14$ were anti-topo I antibody-positive (Figure 2). The higher the skin score, the higher the probability of being anti-topo I-positive $(P<0.001)$.

Patients with anti-topo I antibodies had a higher prevalence of tendon friction rubs that could be interpreted as a sign of active disease. Furthermore, anti-topo I antibodies were associated with contractures and electrocardiogram changes (Table 3).

\section{Anti-topoisomerase I-positive patients showed a higher disease activity and mortality}

The SSc activity score was available for 266 out of 280 patients. Patients with anti-topo I antibodies as detected by LIA revealed a higher disease activity score when compared with antibody-negative SSc patients (Table 3). There was also a weak correlation between disease activity and signal strengths determined by LIA $(P=0.01, \rho=0.234)$. The correlation was slightly better for patients with diffuse SSc. Here, the Spearman's rank correlation coefficient was 0.237 for the correlation between disease activity and LIA data (data not shown).
After serum withdrawal for antibody detection, all patients were followed up for a mean period of 24.6 months. During this period, 16 SSc patients of our cohort died on average 7 years after diagnosis - 14 patients from SSc-associated complications, two patients from malignancies. In diffuse SSc patients, there was a low overall cumulative mortality. Eight patients of the diffuse SSc group died, and five of these patients were anti-topo I antibody-positive, revealing no significant differences between the anti-topo I-positive and antitopo I-negative patients. The presence of anti-topo I antibodies was important for early mortality $(P=0.03$; Figure $3 a$ ), however, especially when high signal strengths above 150 units were studied in the diffuse subset of SSc patients $(P=0.003$; Figure 3b).

\section{Signal strengths in line immunoblot assay correlated with the extent of skin and lung fibrosis}

LIA signals correlated to the extent of skin and lung fibrosis. There was a weak but significant correlation between the LIA signal strengths and mRSS values $(P=0.01, \rho=0.413)$. Furthermore, the signal strengths correlated negatively with the predicted DLCO values by a single breath $(P=0.01, \rho=$ $0.219)$ and with the predicted FVC values $(P=0.01, \rho=$ $0.215)$. 
Figure 2

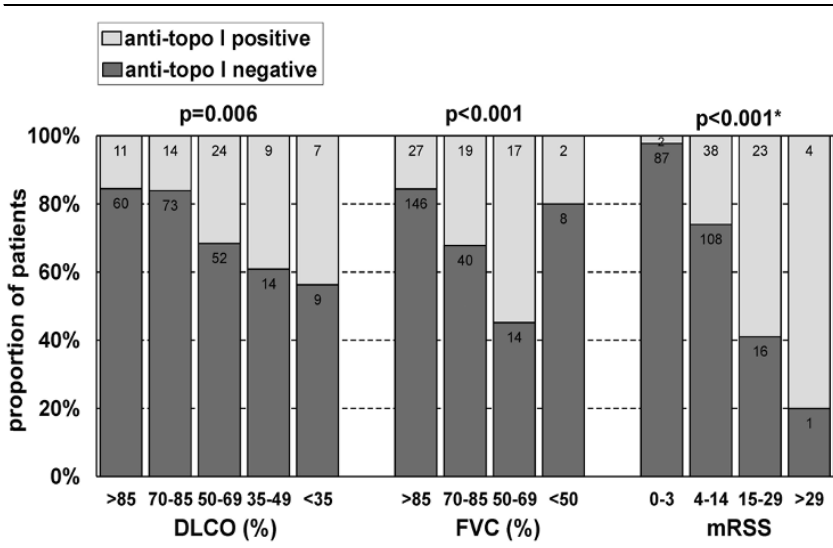

Detection of anti-topoisomerase I antibodies by line immunoblot assay. Detection of anti-topoisomerase I (anti-topo I) antibodies by line immunoblot assay with respect to different levels of predicted diffusion capacity (DLCO) by a single breath (\%), predicted forced vital capacity (FVC) (\%) and modified Rodnan skin score (mRSS) values in 280 patients with systemic sclerosis (SSc) and diseases related to SSc. Numbers in bars, absolute counts. $P$ values are results of the chisquare test. *For the chi-square test, because of the low case numbers in the last group, the last two mRSS groups were joined to hold the test assumptions (maximum $20 \%$ of cells have expected cell count $<5$ ).

\section{Discussion}

The present study demonstrates the diagnostic significance of anti-topo I autoantibodies for clinical assessment of SSc patients in a large monocentric cohort. Antibody detection was performed by means of two commercial test systems, LIA and ELISA, both of which equally identified patients with active diffuse SSc with a higher burden of skin and lung fibrosis, electrocardiogram changes, digital ulcers, and contractures. Importantly, detected signal strengths correlated with skin score values, lung function parameters, and disease activity. We also showed a negative correlation between the predicted DLCO levels and the levels of anti-topo I antibodies, to our knowledge not identified by other studies, suggesting that the decline of DLCO in anti-topo I-positive patients is associated with lung fibrosis. Additionally, high signal strengths were associated with increased mortality in the diffuse SSc patients, indicating the potential of the applied test systems to identify patients with a more severe form of diffuse SSc.

Our cohort is part of the EUSTAR network and, compared with the EUSTAR patients, our anti-topo I-positive SSc patients showed similar clinical data, such as mean mRSS, age, disease duration, frequency of lung fibrosis, pulmonary arterial hypertension, and percentages of predicted DLCO [16]. In line with this correlation, the frequency of anti-topo I-positive patients was nearly identical - with $60.4 \%$ in our cohort compared with $60.8 \%$ in the EUSTAR cohort. For the EUSTAR database, the test used to identify anti-topo I antibodies is not defined and each centre is free to use the method most available. Our results support the conclusion that LIA or ELISA can be used similarly to other assays without loss of information.
Furthermore, the similarities of our cohort with the European databank suggest that our cohort of SSc patients is representative for European SSc patients. Nevertheless, the frequency of anti-topo I antibodies is much higher than found in other cohorts, such as the Pittsburgh cohort with a frequency of $26 \%$ among diffuse SSc patients [6]. In their cohort, anti-topo I antibodies were detectable in $25 \%$ of the patients with limited SSc [1], representing a contrast to the low frequency of $6.2 \%$ in our 113 limited SSc patients.

In our monocentric cohort, most patients have suffered from SSc for longer than 6 years (Table 1). At the time of the present study, patients were already classified according to their maximal skin sclerosis. According to the DNSS and EUSTAR assessment strategy, the subclassification does not change - independent of the current distribution of skin sclerosis. Since LeRoy classification criteria are easily applicable to the extremes of limited SSc and diffuse SSc, there are patients between these typical SSc features. In those patients between these features, access to antibody levels, disease course, and organ manifestations could be helpful to classify the disease. We assume that the number of patients with current limited SSc is higher even in the anti-topo I antibody-positive patients. This could explain differences in the prevalence of anti-topo I antibodies among limited SSc patients. Furthermore, differences in the subclassification, antibody status, and clinical associations could be confounded by different ethnic backgrounds $[11,29]$. In our study, the population of anti-topo I-positive patients was almost homogeneously Caucasian. Only six patients in the anti-topo I-negative cohort had a different genetic background. By deleting these genetically different patients, the results of statistical analyses did not become different. Taking these factors together, in the present study the classification of the SSc patients into diffuse SSc and limited SSc was not done exclusively by measuring the current distribution of skin sclerosis. We also were aware of the autoantibody findings and the previous course of disease that can be critically discussed. A limitation of the present study is therefore a possible overestimation of diffuse SSc patients in our cohort. As recently published by our group analysing a large cohort of more than 1,000 German SSc patients, however, skin sclerosis is only of limited value to identify SSc patients with severe organ manifestations [30].

As shown by other studies using much larger cohorts, antitopo I antibodies characterize patients with a higher extension of skin and lung fibrosis $[1,6,13,16]$. Other studies have also identified associations between the presence of anti-topo I antibodies with digital ulcers and with arthritis not confirmed by other works $[6,30,31]$. Furthermore, the assessment of the organ involvement seems to be crucial and often varies in different studies. When cardiac involvement is studied, different definitions are used. One group defined cardiac involvement by the presence of pericarditis or conduction disturbances of nonischaemic origin, and identified an association between 


\section{Survival and anti-topo I ab Survival and signal strengths}

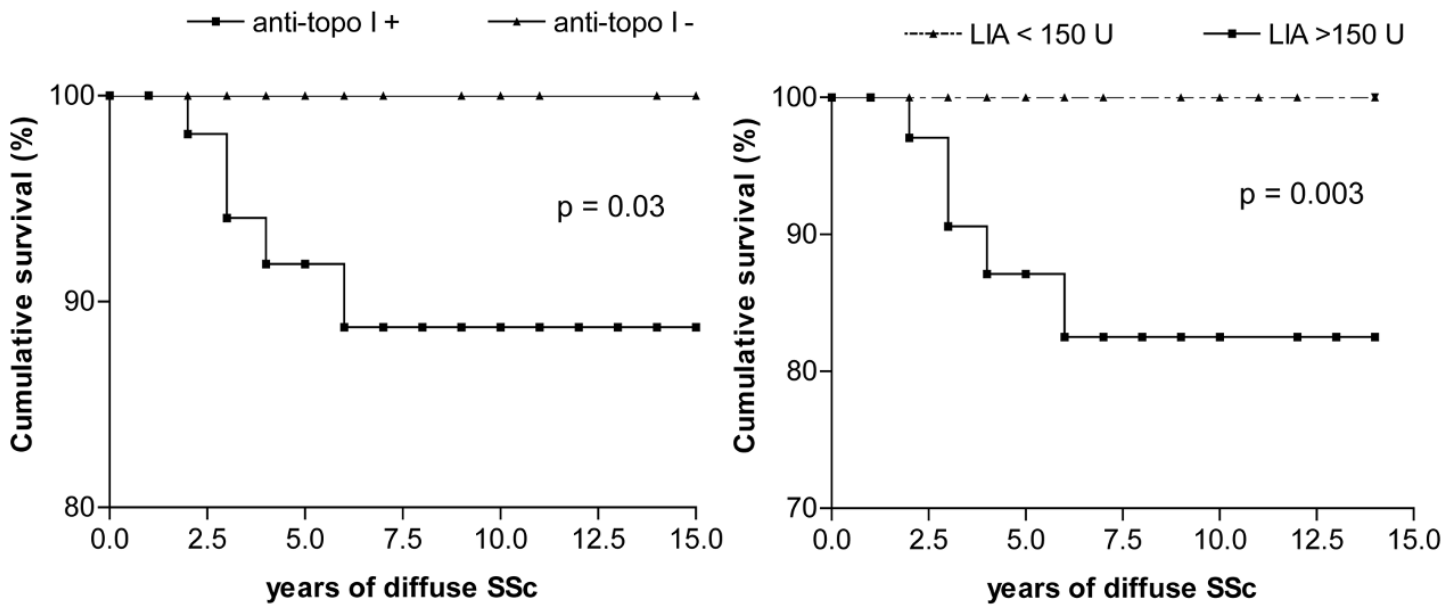

\begin{tabular}{|l|c|c|c|c|}
\hline Years after diagnosis & 0 & 5 & 10 & 15 \\
\hline LIA Anti-topo I+ & 58 & 32 & 16 & 4 \\
\hline LIA Anti-topo - & 38 & 24 & 12 & 7 \\
\hline LIA $>150 \mathrm{U}$ & 38 & 21 & 10 & 5 \\
\hline LIA $<150 \mathrm{U}$ & 58 & 35 & 18 & 8 \\
\hline
\end{tabular}

Cumulative survival from time of diagnosis related to the presence of anti-topoisomerase I antibodies. Cumulative survival rates from the time of diagnosis related to the presence of anti-topoisomerase I antibodies (anti-topo I ab) in our cohort of 96 patients with diffuse systemic sclerosis (SSc) as detected by line immunoblot assay (LIA). Signal strengths above 150 units were associated with increased mortality $(P=0.003)$.

cardiac involvement and the presence of anti-topo I antibodies - supporting the results from our study [5]. Another group defined heart involvement by symptomatic pericarditis, congestive heart failure, or arrhythmias requiring treatment and did not find any association with the serological status [6]. Since the assessment of subjective symptoms can be influenced by different reasons, and - as shown especially for the therapy of arrhythmias - guidelines and treatment modalities may vary, there is a need to use objective parameters to identify involvement of specific organs. International consensus criteria for assessment would be important for the evaluation of a potential biomarker and also to identify differences in the prevalence of organ involvement, but have so far not been established. In this setting, large monocentric cohorts with a homogeneous assessment could provide the most valuable data.

Comparing the cumulative survival in different studies, there was a high cumulative survival among our diffuse SSc patients and also among the anti-topo I-positive diffuse SSc patients in our cohort (about 92\% in 5 years; Figure 3).

In an Italian cohort, cumulative 5-year survival from the first diagnosis was a little bit lower, with about $86 \%$ in anti-topo Ipositive patients. The prevalence of organ involvement was similar to our group; however, by providing lung function or mRSS data, the extent of skin and lung fibrosis was not specified, making a comparison between the groups difficult [14]. In the Pittsburgh group, the cumulative 5-year survival from the initial visit of 102 anti-topo I-positive patients was about $70 \%$. When the 68 diffuse anti-topo I-positive patients were studied, cumulative survival from the first symptom based on disease classification and autoantibody status was about 82\% [6]. The high mean mRSS of 32 indicates an SSc cohort with severe SSc that seems to be not representative for cohorts found in Europe among the anti-topo I-positive patients, which was also suggested by other workers [32]. The EUSTAR database will provide the mortality found in SSc patients in Europe, providing a valuable tool to identify differences among the European countries and different ethnic groups.

For the evaluation of the diagnostic impact of a biomarker such as anti-topo I antibodies and for the comparison with other studies, the interval between antibody detection and clinical assessment may be important. Sato and colleagues also described a correlation between the levels of anti-topo I antibodies and mRSS values and the predicted vital capacities in a small number of anti-topo I-positive patients $(n=30)$ [30]. The correlations were much better than in our cohort. In con- 
trast to our study, the authors did not provide information about the time point for the assessment of clinical data. For a progressive disease such as SSc, the degree of correlation between anti-topo I antibodies and the cumulative mRSS and FVC may be better if patients with more advanced disease were assessed. On the other hand, as shown by others, loss of anti-topo I reactivity during the course of the disease may occur in up to $20 \%$ of the patients [12], suggesting that a simultaneous assessment could be important.

Using the mRSS as a surrogate parameter to measure disease activity, other studies could also show an increased disease activity in anti-topo I-positive patients [3]. The mRSS, however, is only one parameter in the different disease activity scores used $[17,18]$. In the present study we have shown for the first time a weak but significant correlation between disease activity scores and the levels of anti-topo I antibodies or the signal strengths obtained by LIA or ELISA. For the assessment of the disease activity, we have used the EUSTAR activity score suggesting that this method is a valuable test for the assessment of disease activity, as supported by Valentini and colleagues [33].

In conclusion, anti-topo I antibodies represent an appropriate and important parameter for the diagnosis and risk assessment of SSc patients. As exemplified here for the evaluation of anti-topo I antibodies, a precise characterization of patients and international consensus criteria for the assessment of patients are crucial and may influence the results. Where these tools are not available, large monocentric cohorts may provide a valuable tool for the evaluation of biomarkers.

\section{Conclusion}

Anti-topo I autoantibodies can provide important information for the diagnosis of SSc patients. For the detection of these antibodies, carefully validated monospecific test methods, such as the LIA and ELISA applied in the present study, are equally competent and allow risk assessment due to the correlation between signal strength and severity of fibrotic manifestations, disease activity and prognosis.

The evaluation of potential biomarkers would profoundly benefit from the development and implementation of international consensus criteria for the classification of SSc patients. In this respect, large monocentric cohorts with a standardized assessment may provide the most valuable approach at present.

\section{Competing interests}

GR received lecturer's fees from EUROIMMUN AG to show the data at the 'Eurodoctor' meeting in Brussels. $\mathrm{KH}$ was invited by EUROIMMUN AG to participate in a national meeting to show the results of the study. After finishing the study, $\mathrm{KH}$ received a grant from EUROIMMUN AG for another scien- tific work. All the other authors declare that they have no competing interests.

\section{Authors' contributions}

$\mathrm{KH}$ performed preclinical analyses, statistics, graphics and has partially written the manuscript. CD, AJ and WM developed the LIA and ELISA and performed the tests. CSB and $\mathrm{MB}$ provided the clinical data together with GR. MB corrected and helped to write the manuscript. $\mathrm{DH}$ helped with the statistics and guided $\mathrm{KH}$ to perform the right tests. $\mathrm{KE}$ and $\mathrm{FH}$ as well as GRB discussed the data with the last author and gave intellectual contributions. KE provided sera for the analysis. WS organized all cooperation with EUROIMMUN AG and provided intellectual contributions. GR, as the last and responsible author, initiated this study and controlled the work. GR wrote and reviewed the manuscript.

\section{Acknowledgements}

The present study was supported by the BMBF-sponsored German Network of Systemic Sclerosis BMBF Fkz 01 GM 0310 (C2, C6, TP6) and by the European Scleroderma Trial and Research network. EUROIMMUN AG performed the detection of the antibodies without any knowledge of the clinical data.

\section{References}

1. Perera A, Fertig N, Lucas M, Rodriguez-Reyna TS, Hu P, Steen VD, Medsger TA Jr: Clinical subsets, skin thickness progression rate, and serum antibody levels in systemic sclerosis patients with anti-topoisomerase I antibody. Arthritis Rheum 2007, 56:2740-2746.

2. Scussel-Lonzetti L, Joyal F, Raynauld JP, Roussin A, Rich E, Goulet JR, Raymond Y, Senécal JL: Predicting mortality in SSc. Analysis of a cohort of 309 French Canadian patients with emphasis on features at diagnosis as predictive factors for survival. Medicine 2002, 81:154-167.

3. Hu PQ, Fertig N, Medsger TA Jr, Wright TM: Correlation of serum anti-DNA topoisomerase I antibody levels with disease severity and activity in systemic sclerosis. Arthritis Rheum 2003 , 48:1363-1373.

4. Diot E, Giraudeau B, Lemarié E, Degenne D, Ritz L, Guilmot JL, Lemarié E: Is anti-topoisomerase I a serum marker of pulmonary involvement in systemic sclerosis? Chest 1999, 116:715-720.

5. Weiner ES, Earnshaw WC, Senecal JL, Bordwell B, Johnson P, Rothfield NF: Clinical associations of anticentromere antibodies and antibodies to topoisomerase I: a study of $\mathbf{3 5 5}$ patients. Arthritis Rheum 1988, 31:378-385.

6. Steen VD, Powell DL, Medsger TA Jr: Clinical correlations and prognosis based on serum autoantibodies in patients with SSc. Arthritis Rheum 1988, 31:196-203.

7. Bizzaro N, Tonutti E, Villalta D, Bassetti D, Tozzoli R, Manoni F, Pirrone S, Piazza A, Rizzotti P, Pradella M: Sensitivity and specificity of immunological methods for the detection of anti-topoisomerase I (Scl70) autoantibodies: results of a multicenter study. The Italian Society of Laboratory Medicine Study Group on the Diagnosis of Autoimmune diseases. Clin Chem 2000, 46:1681-1685.

8. Tamby MC, Bussone G, Mouthon L: Antitopoisomerase 1 antibodies in systemic sclerosis: how to improve the detection? Ann NY Acad Sci 2007, 1109:221-228.

9. Vos PA, Bast EJ, Derksen RH: Cost-effective detection of nonantidouble-stranded DNA antinuclear antibody specificities in daily clinical practice. Rheumatology (Oxford) 2006, 45:629-635.

10. Maclachlan D, Vogt P, Wu X, Rose L, Tyndall A, Hasler P: Comparison between line immunoassay (LIA) and enzyme-linked immunosorbent assay (ELISA) for the determination of antibodies to extractable nuclear antigenes (ENA) with reference 
to other laboratory results and clinical features. $Z$ Rheumatol 2002, 61:534-544.

11. Kuwana M, Kaburaki J, Arnett FC, Howard RF, Medsger TA Jr, Wright TM: Influence of ethnic background on clinical and serological features in patients with SSc and Anti-DNA Topoisomerase I antibody. Arthritis Rheum 1999, 42:465-474.

12. Kuwana M, Kaburaki J, Mimori T, Kawakami Y, Tojo T: Longitudinal analysis of autoantibody response to topoisomerase I in SSc. Arthritis Rheum 2000, 43:1074-1084.

13. Kuwana M, Kaburaki J, Okano Y, Tojo T, Homma M: Clinical and prognostic associations based on serum antinuclear antibodies in Japanese patients with systemic sclerosis. Arthritis Rheum 1994, 37:75-83.

14. Ferri C, Valentini G, Cozzi F, Sebastiani M, Michelassi C, La Montagna G, Bullo A, Cazzato M, Tirri E, Storino F, Giuggioli D, Cuomo G, Rosada M, Bombardieri S, Todesco S, Tirri G, Systemic Sclerosis Study Group of the Italian Society of Rheumatology (SIRGSSSc): Systemic sclerosis. Demographic, clinical, and serologic features and survival in 1012 Italian patients. Medicine 2002, 81:139-153.

15. LeRoy EC, Black C, Fleischmajer R, Jablonska S, Krieg T, Medsger TA Jr, Rowell N, Wollheim F: Scleroderma (systemic sclerosis): classification, subsets and pathogenesis. J Rheumatol 1988, 15:202-205.

16. Walker UA, Tyndall A, Czirják L, Denton C, Farge-Bancel D, KowalBielecka O, Müller-Ladner U, Bocelli-Tyndall C, Matucci-Cerinic M: Clinical risk assessment of organ manifestations in systemic sclerosis: a report from the EULAR Scleroderma Trials and Research group database. Ann Rheum Dis 2007, 66:754-763.

17. Valentini G, Bencivelli W, Bombardieri S, D'Angelo S, Della Rossa A, Silman AJ, Black CM, Czirjak L, Nielsen H, Vlachoyiannopoulos PG: European Scleroderma Study Group to define disease activity criteria for systemic sclerosis. III. Assessment of the construct validity of the preliminary activity criteria. Ann Rheum Dis 2003, 62:901-903.

18. Medsger TA Jr, Silman AJ, Steen VD, Black CM, Akesson A, Bacon PA, Harris CA, Jablonska S, Jayson MI, Jimenez SA, Krieg T, Leroy EC, Maddison PJ, Russell ML, Schachter RK, Wollheim FA, Zacharaie $\mathrm{H}:$ A disease severity scale for systemic sclerosis: development and testing. J Rheumatol 1999, 26:2159-2167.

19. Czirják L, Nagy Z, Aringer M, Riemekasten G, Matucci-Cerinic M, Furst DE, EUSTAR: The EUSTAR model for teaching and implementing the modified Rodnan skin score in systemic sclerosis. Ann Rheum Dis 2007, 66:966-969.

20. Hunzelmann N, Genth E, Krieg T, Lehmacher W, Melchers I, Meurer M, Moinzadeh P, Müller-Ladner U, Pfeiffer C, Riemekasten G, Schulze-Lohoff E, Sunderkoetter C, Weber M, Worm M, Klaus $P$, Rubbert A, Steinbrink K, Grundt B, Hein R, ScharffetterKochanek K, Hinrichs R, Walker K, Szeimies RM, Karrer S, Müller A, Seitz C, Schmidt E, Lehmann P, Foeldvári I, Reichenberger F, et al.: The registry of the German Network for Systemic Scleroderma: frequency of disease subsets and patterns of organ involvement. Rheumatology (Oxford) 2008, 47:1185-1192.

21. American Rheumatism Association Subcommittee for Scleroderma Criteria, Diagnostic and Therapeutic Criteria committee: Preliminary criteria for the classification of systemic sclerosis (scleroderma). Arthritis Rheum 1980, 23:581-590.

22. Bennett RM: Scleroderma overlap syndromes. Rheum Dis Clin North Am 1990, 16:185-198.

23. Poormoghim H, Lucas M, Fertig N, Medsger TA Jr: Systemic sclerosis sine scleroderma: demographic, clinical, and serologic features and survival in forty-eight patients. Arthritis Rheum 2000, 43:444-451.

24. Alarcon GS: Unclassified or undifferentiated connective tissue disease. Baillieres Best Pract Res Clin Rheumatol 2000, 14:125-137.

25. Furst DE, Clements PJ, Steen VD, Medsger TA Jr, Masi AT, D'Angelo WA, Lachenbruch PA, Grau RG, Seibold JR: The modified Rodnan skin score is an accurate reflection of skin biopsy thickness in SSc. J Rheumatol 1998, 25:84-88.

26. Rodnan RP, Myerowitz RL, Justh GO: Morphologic changes in digital arteries of SSc and RP. Medicine 1980, 58:393-408.

27. Hildebrandt S, Weiner ES, Senecal JL, Noell GS, Earnshaw WC, Rothfield NF: Autoantibodies to topoisomerase I (Scl-70): analysis by gel diffusion, immunoblot, and enzyme-linked immunosorbent assay. Clin Immunol Immunopathol 1990, 57:399-410.
28. Hunzelmann N, Genth E, Krieg T, Meurer M, Melchers I, Moinzadeh P, Pfeiffer C, Riemekasten G, Schulze-Lohoff E, Sunderkoetter C, Müller-Ladner U: [Organ-specific diagnosis in patients with systemic sclerosis: recommendations of the German Network for Systemic Sclerosis (DNSS)]. Z Rheumatol 2008, 67:334-340. Article in German

29. Koenig M, Dieudé M, Senécal JL: Predictive value of antinuclear autoantibodies: the lessons of the systemic sclerosis autoantibodies. Autoimmun Rev 2008, 7:588-593.

30. Sato S, Hamaguchi Y, Hasegawa M, Takehara K: Clinical significance of anti-topoisomerase I antibody levels determined by ELISA in systemic sclerosis. Rheumatology (Oxford) 2001, 40:1135-1140.

31. Hanitsch LG, Burmester GR, Witt C, Hunzelmann N, Genth E, Krieg T, Lehmacher W, Melchers I, Meurer M, Müller-Ladner U, Schulze-Lohoff E, Becker M, Sunderkoetter C, Worm M, Klaus P, Rubbert A, Steinbrink K, Grundt B, Hein R, Scharffetter-Kochanek K, Hinrichs R, Walker K, Szeimies R-M, Karrer S, Müller A, Seitz C, Schmidt E, Lehmann P, Foeldvári I, Riemekasten G, et al:: Skin sclerosis is only of limited value to identify SSc patients with severe manifestations - an analysis of a distinct patient subgroup of the German Systemic Sclerosis Network (DNSS) Register. Rheumatology (Oxford) 2009, 48:70-73.

32. Meyer OC, Fertig N, Lucas M, Somogyi N, Medsger TA Jr: Disease subsets, antinuclear antibody profile, and clinical features in 127 French and 247 US adult patients with systemic sclerosis. J Rheumatol 2007, 34:104-109.

33. Valentini G, Della Rossa A, Bombardieri S, Bencivelli W, Silman AJ, D'Angelo S, Cerinic MM, Belch JF, Black CM, Bruhlmann P, Czirják L, De Luca A, Drosos AA, Ferri C, Gabrielli A, Giacomelli R, Hayem G, Inanc M, McHugh NJ, Nielsen H, Rosada M, Scorza R, Stork J, Sysa A, Hoogen FH van den, Vlachoyiannopoulos PJ: European multicentre study to define disease activity criteria for systemic sclerosis. II. Identification of disease activity variables and development of preliminary activity indexes. Ann Rheum Dis 2001, 60:592-598. 